\title{
Algorithm-based troubleshooting to manage bleeding during thoracoscopic anatomic pulmonary resection
}

\author{
Hitoshi Igai, Mitsuhiro Kamiyoshihara, Ryohei Yoshikawa, Fumi Ohsawa, Tomohiro Yazawa, \\ Natsumi Matsuura \\ Department of General Thoracic Surgery, Japanese Red Cross Maebashi Hospital, 389-1 Asakura-cho, Maebashi, Gunma 371-0811, Japan \\ Contributions: (I) Conception and design: H Igai; (II) Administrative support: H Igai; (III) Provision of study materials or patients: All authors; (IV) \\ Collection and assembly of data: All authors; (V) Data analysis and interpretation: H Igai; (VI) Manuscript writing: All authors; (VII) Final approval \\ of manuscript: All authors. \\ Correspondence to: Hitoshi Igai, MD, PhD. 389-1 Asakura-cho, Maebashi, Gunma 371-0811, Japan. Email: hitoshiigai@gmail.com.
}

\begin{abstract}
Background: Few studies have reported on the effects of intraoperative complications, such as vessel injury, during thoracoscopic anatomic pulmonary resection. We evaluated intraoperative vessel injury and assessed troubleshooting methods for thoracoscopic anatomic pulmonary resection.

Methods: A total of 378 patients underwent thoracoscopic anatomic pulmonary resection between April 2012 and March 2018, 40 of whom were identified as having an intraoperative vessel injury. In our department, we treat significant bleeding based on the algorithm shown in Figure 1. We analyzed the injured vessels and hemostatic procedures employed and compared perioperative outcomes in patients with $(\mathrm{n}=40)$ or without ( $n=338$ ) a vessel injury. Additionally, we examined the data on a year-by-year basis from April 2012, and perioperative results were compared in each year.

Results: The vessel injured was a branch of the pulmonary artery in 22 cases (55\%). Hemostasis was achieved by applying a thrombostatic sealant in 26 cases $(65 \%)$. Although patients without a vessel injury had a shorter operation time, less intraoperative blood loss, and shorter duration of chest tube drainage, no significant differences in the length of postoperative hospitalization or morbidity were observed. The occurrence rate of significant intraoperative bleeding in the last year measured was similar to that in the first year measured.

Conclusions: Thoracoscopic anatomic pulmonary resection is feasible and safe if the surgeon performs appropriate hemostasis, although vascular hazards might be inherent during thoracoscopic anatomic pulmonary resection, regardless of the surgeon's experience.
\end{abstract}

Keywords: Algorithm; troubleshooting; intraoperative bleeding; thoracoscopy; pulmonary resection

Submitted Jul 29, 2019. Accepted for publication Oct 10, 2019.

doi: $10.21037 /$ jtd.2019.10.72

View this article at: http://dx.doi.org/10.21037/jtd.2019.10.72

\section{Introduction}

Thoracoscopic anatomic pulmonary resection is applied worldwide because many authors have reported better perioperative results compared to using the thoracotomy approach over the last few years (1-4). The thoracoscopic approach is considered less invasive and has several advantages, including less postoperative pain, less morbidity, reduced duration of postoperative chest drainage, and shorter hospitalization, which indicates more rapid postoperative recovery and reduced medical costs. Moreover, several reports have described that the thoracoscopic approach is not inferior to a thoracotomy approach for early-stage lung cancer patients in terms of oncological prognosis (1). These results indicate that the thoracoscopic approach is the preferred approach for earlystage lung cancer.

It is important to manage significant intraoperative 
bleeding appropriately to ensure the safety of the operation and to minimize the conversion rate to a thoracotomy. Therefore, thoracic surgeons should have the appropriate hemostatic procedural skill in thoracoscopic surgery because inappropriate management can result in catastrophic bleeding, which sometimes leads to a lifethreatening condition for patients. Moreover, as the thoracoscopic approach has some limitations in terms of ease of moving forceps and surgical view, the management of a vascular injury during thoracoscopic surgery is much more delicate than during a thoracotomy (5). Therefore, simple techniques should be used to achieve appropriate hemostasis during the thoracoscopic approach.

Although several authors have shown how to manage intraoperative bleeding during thoracoscopic pulmonary resection, there are no standardized hemostatic procedures (6-13). Therefore, our team focused on the actual surgical techniques to manage intraoperative vessel injury and created an algorithm for managing significant intraoperative bleeding during thoracoscopic surgery to standardize the hemostatic procedures. Here, we review the surgical techniques used to achieve hemostasis during thoracoscopic surgery and investigate how to manage intraoperative bleeding appropriately.

\section{Methods}

This study was approved by the Japanese Red Cross Maebashi Hospital Institutional Review Board (No. 2019-40).

A total of 378 patients underwent thoracoscopic anatomic pulmonary resection between April 2012 and March 2018, 40 of whom were identified as having an intraoperative vessel injury. We retrospectively analyzed the injured vessels and hemostatic procedures employed and compared the patient characteristics and perioperative outcomes in patients with $(n=40)$ or without $(n=338)$ a vessel injury. Additionally, we examined the data on a yearby-year basis starting from April 2012, and perioperative results were compared each year. Significant vessel injury was defined as bleeding that needed compression times of $>30 \mathrm{~s}$ for hemostasis.

Thoracoscopic anatomic pulmonary resection was performed under general anesthesia using one-lung ventilation with the patient in the lateral decubitus position. We placed a monitor above the head of the patient and all procedures were performed under the monitor. A 10- or 5 -mm flexible type thoracoscope was used in all patients. Our thoracoscopic anatomic pulmonary resection uses three or four ports. A $3-\mathrm{cm}$ access incision was placed in the fourth or fifth intercostal space on the anterior axillary line. The thoracoscopic port was placed in the sixth or seventh intercostal space on the posterior axillary line. One or two additional ports were placed for surgical instruments in the sixth intercostal space on the anterior axillary line or the sixth or seventh intercostal space just beside the scapular angle. The access incision and additional ports for surgical instruments were covered with a size XXS wound retractor (Alexis Wound Retractor; Applied Medical, Rancho Santa Margarita, CA, USA). Large vessels and bronchi were divided with a stapler. Small-caliber vessels were divided with an energy device or scissors after ligation. The specimen was placed in an endovascular bag and retrieved through the access port after we completed the pulmonary resection. If necessary, the access incision was lengthened depending on the size of the specimen. We never used a rib-spreader in any situation. A systemic lymphadenectomy was performed for patients with primary lung cancer undergoing lobectomy. The hilar lymph nodes were sampled in patients with primary lung cancer undergoing a segmentectomy. No lymphadenectomy was performed in patients with metastatic lung cancer or benign diseases.

In our department, we treat significant bleeding based on the algorithm shown in Figure 1. In the algorithm, the bleeding point on the intrathoracic vessel was initially compressed with adjacent lung parenchyma or a cotton stick. In cases of bleeding from a pulmonary artery, thrombostatic sealant, chiefly TachoSil (CSL Behring, Tokyo, Japan), was subsequently introduced into the thorax through a port and attached to the bleeding point to avoid intraoperative or postoperative re-bleeding after hemostasis. If we did not achieve hemostasis with compression, we attempted to achieve hemostasis using a TachoSil attachment. We initially cut the sealant into a $7 \times 7-\mathrm{mm}$ square, and then attached a piece of sealant to the top of a cotton stick with medical jelly for smooth insertion into the thorax and application to the bleeding point. A second or third piece of sealant was added if hemostasis was not achieved using the first attachment. In the case of bleeding from other vessels, including the pulmonary vein, bronchial artery, or azygos vein, clipping or suturing was attempted after compression. A thrombostatic sealant was attached to the bleeding point for hemostasis when it was difficult to achieve hemostasis by clipping or suturing. A thoracoscopic approach was converted to a thoracotomy when total blood loss reached $>600 \mathrm{~mL}$ or the operating surgeon considered that vascular clamps were required to control massive 


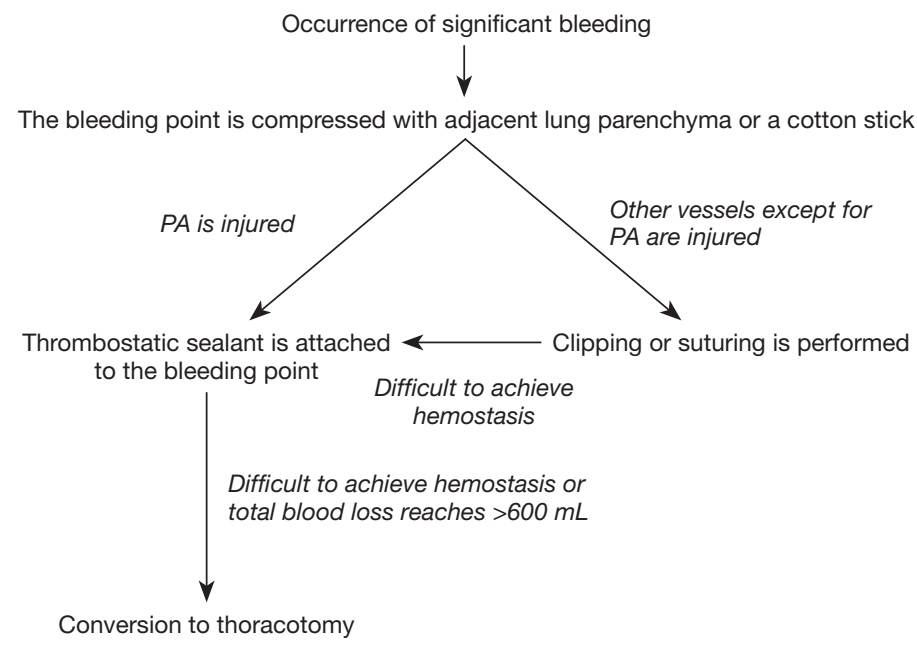

Figure 1 The algorithm used to manage significant intraoperative bleeding during thoracoscopic surgery in our department. As an initial treatment, we compress the bleeding point using adjacent lung tissue or a cotton stick. After compression, we attach a thrombostatic sealant to the bleeding point to ensure hemostasis when pulmonary artery branches are injured. If intrathoracic vessels other than the pulmonary artery are injured, the injury point is clipped or sutured. When we have difficulty achieving hemostasis or total blood loss reaches $>600 \mathrm{~mL}$, the thoracoscopic surgery is converted to a thoracotomy.

Table 1 Comparison of the characteristics of patients with and without vessel injuries

\begin{tabular}{lccc}
\hline Characteristics & $\begin{array}{c}\text { Vessel injury } \\
\text { group }(\mathrm{n}=40)\end{array}$ & $\begin{array}{c}\text { Non-vessel injury } \\
\text { group }(\mathrm{n}=338)\end{array}$ & $\mathrm{P}$ value \\
\hline Mean age (years) & $70 \pm 10$ & $70 \pm 11$ & 0.89 \\
Sex & & & 0.059 \\
Male & $30(75.0)$ & $198(58.6)$ & \\
Female & $10(25.0)$ & $140(41.4)$ & \\
Targeted lung area for & & & \\
resection & & & \\
Right upper lobe & $13(32.5)$ & $103(30.5)$ & \\
Right middle lobe & $2(5.0)$ & $22(6.5)$ & \\
Right lower lobe & $6(15.0)$ & $73(21.6)$ & \\
Left upper lobe & $13(32.5)$ & $70(20.7)$ & \\
Left lower lobe & $6(15.0)$ & $70(20.7)$ & \\
Disease necessitating resection & & \\
Primary lung cancer & $34(85.0)$ & $279(82.5)$ & \\
Metastatic lung & $4(10.0)$ & $30(8.9)$ & \\
cancer & & & \\
Benign disease & $2(5.0)$ & $29(8.6)$ & \\
Surgical procedure & & $57(16.9)$ & \\
Bilobectomy & $0(0)$ & & \\
Lobectomy & $33(82.5)$ & $280(82.8)$ & \\
Segmentectomy & $7(17.5)$ & 57.8 & \\
\hline
\end{tabular}

Except for mean age, data are reported as the numbers of patients with percentages in parentheses. bleeding.

To compare patient characteristics and perioperative results between patients with and without a vessel injury, statistical analyses using the independent $t$-test or Fisher's exact test were performed. Differences were considered significant at $\mathrm{P}<0.05$. Calculations and statistical tests were performed using EZR software (Saitama Medical Center, Jichi Medical University; http://www.jichi.ac.jp/saitama-sct/ SaitamaHP.files/statmed EN.html; Kanda, 2012).

\section{Results}

Table 1 shows a comparison of the characteristics of patients with $(n=40)$ and without $(n=338)$ vessel injuries. No significant differences were observed between the two groups in terms of mean age $(70 \pm 10$ vs. $70 \pm 11$ years, respectively; $\mathrm{P}=0.89$ ), sex ratio (10 females and 30 males $v s .140$ females and 198 males, respectively; $\mathrm{P}=0.059$ ), targeted lung area for resection (right upper lobe, right middle lobe, right lower lobe, left upper lobe, and left lower lobe: 13, 2, 6, 13, and 6 patients vs. 103, 22, 73, 70, and 70 patients, respectively; $\mathrm{P}=0.49$ ), diseases necessitating resection (primary lung cancer, metastatic lung cancer, benign diseases: 34,4 , and 2 patients vs. 279, 30, and 29 patients, respectively; $\mathrm{P}=0.8$ ), and surgical procedures (33 lobectomies and 7 segmentectomies vs. 1 bilobectomy, 280 lobectomies, and 
Table 2 Details of the vessel injuries and the procedures used for hemostasis

\begin{tabular}{lc}
\hline Variables & No. (\%) \\
\hline Injury points & $22(55.0)$ \\
PA branches & $13(32.5)$ \\
PV branches & $3(7.5)$ \\
Bronchial artery & $2(5.0)$ \\
Azygos vein & \\
Hemostatic procedure & $26(65.0)$ \\
Thrombostatic sealant & $10(25.0)$ \\
Compression & $2(5.0)$ \\
Stapling & $1(2.5)$ \\
Ligation & $1(2.5)$ \\
Clipping & \\
Recovery approach & $35(87.5)$ \\
Thoracoscopy & $5(12.5)$ \\
Thoracotomy & \\
\hline
\end{tabular}

PA, pulmonary artery; PV, pulmonary vein.

Table 3 Comparison of perioperative results in patients with $(n=40)$ and without $(\mathrm{n}=338)$ vessel injuries

\begin{tabular}{lccc}
\hline Variable & $\begin{array}{c}\text { Vessel injury } \\
\text { group }(\mathrm{n}=40)\end{array}$ & $\begin{array}{c}\text { Non-vessel } \\
\text { injury group } \\
(\mathrm{n}=338)\end{array}$ & P value \\
\hline Operation time (min) & $240 \pm 59$ & $207 \pm 57$ & 0.0005 \\
Blood loss $(\mathrm{mL})$ & $286 \pm 535$ & $53 \pm 109$ & $<0.0001$ \\
$\begin{array}{l}\text { Conversion to } \\
\text { thoracotomy }\end{array}$ & $8(20.0)$ & $15(4.4)$ & 0.0012 \\
$\begin{array}{l}\text { Duration of chest tube } \\
\text { drainage (days) }\end{array}$ & $3.9 \pm 3.6$ & $3 \pm 2.1$ & 0.02 \\
$\begin{array}{l}\text { Length of postoperative } \\
\text { hospital stay (days) }\end{array}$ & $13 \pm 27$ & $8 \pm 17$ & 0.14 \\
Morbidity & $9(22.5)$ & $51(15.1)$ & 0.25 \\
Mortality & $0(0)$ & $0(0)$ & - \\
\hline
\end{tabular}

Data are presented as the means \pm standard deviation or numbers of patients with percentages in parentheses.

57 segmentectomies, respectively; $\mathrm{P}=1$ ).

Table 2 shows the details of the vessel injuries and the procedures used for hemostasis. The vessel injured was a branch of the pulmonary artery in 22 cases $(55.0 \%)$, a
Table 4 Comparison of perioperative results in patients who underwent thoracoscopic anatomic pulmonary resection during the first year (April 2012 to March 2013; n=38) and the last year (April 2017 to March 2018; n=73)

\begin{tabular}{|c|c|c|c|}
\hline Variable & $\begin{array}{c}\text { First year, } \\
n=38\end{array}$ & $\begin{array}{l}\text { Last year, } \\
n=73\end{array}$ & $P$ value \\
\hline Operation time (min) & $244 \pm 57$ & $182 \pm 45$ & $<0.0001$ \\
\hline Blood loss (mL) & $95 \pm 5,149$ & $38 \pm 73$ & 0.0073 \\
\hline Conversion to thoracotomy & $3(7.9)$ & $6(8.2)$ & 0.11 \\
\hline $\begin{array}{l}\text { Intraoperative massive } \\
\text { bleeding }\end{array}$ & $4(10.5)$ & $5(6.8)$ & 0.23 \\
\hline $\begin{array}{l}\text { Duration of chest tube } \\
\text { drainage (days) }\end{array}$ & $3.7 \pm 3.1$ & $2.4 \pm 1.2$ & 0.0032 \\
\hline $\begin{array}{l}\text { Length of postoperative } \\
\text { hospital stay (days) }\end{array}$ & $8.1 \pm 3.3$ & $4.5 \pm 1.9$ & $<0.0001$ \\
\hline Morbidity & $5(13.2)$ & $6(8.2)$ & 0.01 \\
\hline Mortality & $0(0)$ & $0(0)$ & - \\
\hline
\end{tabular}

Data are presented as the means \pm standard deviation or numbers of patients with percentages in parentheses.

branch of the pulmonary vein in 13 cases $(32.5 \%)$, and a different vessel in 5 cases $(12.5 \%)$. The procedure was converted to a thoracotomy in 8 cases $(20 \%)$ to achieve hemostasis. Hemostasis was achieved by applying a thrombostatic sealant in 26 cases $(65.0 \%)$, compression with a cotton stick or adjacent lung parenchyma in 10 cases (25.0\%), and some other method in 4 cases $(10.0 \%)$.

Table 3 shows the perioperative results in patients with $(\mathrm{n}=40)$ and without $(\mathrm{n}=338)$ vessel injuries. Although patients without a vessel injury had a shorter operation time than those with vessel injuries (207 vs. $240 \mathrm{~min}$, respectively; $\mathrm{P}=0.0005)$, and experienced less intraoperative blood loss (53 vs. $286 \mathrm{~mL}$, respectively; $\mathrm{P}<0.0001)$ and shorter duration of chest tube drainage ( 3 vs. 3.9 days, respectively; $\mathrm{P}=0.02$ ), no significant differences were observed in the length of postoperative hospitalization (8vs. 13 days, respectively; $\mathrm{P}=0.14)$ or morbidity rate $(15.1 \%$ vs. $22.5 \%$, respectively; $\mathrm{P}=0.25)$.

Table 4 shows the perioperative results in patients undergoing thoracoscopic anatomic pulmonary resection during the first (April 2012 to March 2013; n=38) and last years (April 2017 to March 2018; n=73) of this study. The occurrence rate of significant intraoperative bleeding in the last year was similar to that during the first year $(6.8 \% v s$. $10.5 \%$, respectively; $\mathrm{P}=0.23$ ), although other perioperative results had improved significantly in that time frame. 


\section{Discussion}

In this study, no significant differences in the length of postoperative hospitalization or morbidity rate were observed between patients with and without a vessel injury, although the patients without a vessel injury experienced a shorter operation time, less intraoperative blood loss, and shorter duration of chest tube drainage. These results indicate that patients with vessel injuries had uneventful postoperative courses, and a longer operation time, more intraoperative blood loss, and a longer duration of chest tube drainage did not affect postoperative hospitalization or morbidity rate significantly. Therefore, it is important to manage significant intraoperative bleeding adequately, although these procedures can take more time and cause additional slight blood loss. We considered that it was necessary to create the algorithm for managing bleeding during thoracoscopic surgery to appropriately troubleshoot the bleeding. Our group has described the algorithm for bleeding during thoracoscopic surgery in previous reports $(14,15)$. In this manuscript, we present the modified algorithm after accumulating more experience. We consider that thoracoscopic anatomic pulmonary resection is safe and feasible if we treat vessel injuries appropriately based on the algorithm.

As a first step to achieving hemostasis, we applied compression to the bleeding point using adjacent lung tissue or a cotton stick. This compression technique is frequently used during thoracoscopic surgery to control bleeding temporally. Other studies have also reported that this compression technique is very important for achieving temporal hemostasis during thoracoscopic surgery (10). Subsequently, we moved to a second step including attachment of the thrombostatic sealant, clipping, or suturing in our algorithm.

Compared to other intrathoracic vessels, such as the pulmonary vein, azygos vein, or bronchial artery, the wall of pulmonary artery is thin and fragile. Therefore, the pulmonary artery is injured more frequently compared to other intrathoracic vessels, and injury can result in catastrophic bleeding if it is not managed adequately and rapidly. Our results revealed that $55 \%$ of the significant intraoperative bleeding during thoracoscopic surgery was caused by injury to pulmonary arterial branches. Moreover, the thin and fragile wall of the pulmonary artery requires a much more delicate treatment of the injury point in addition to a thoracoscopic approach. The bleeding could become catastrophic if the pulmonary artery is torn when suturing the injury point. Several previous reports have also stated that it was very important to treat an injury to the pulmonary artery appropriately during thoracoscopic surgery to avoid catastrophic bleeding $(10,12,13)$. Therefore, we recommend attaching a thrombostatic sealant during thoracoscopic surgery to achieve hemostasis because this technique is quite simple.

Injury to other vessels, including the pulmonary vein, azygos vein, superior vena cava, subclavian artery or vein, and bronchial artery, results in a catastrophic event as well. When we encountered injuries to these vessels, we attempted to achieve hemostasis using suturing or clipping after using the compression technique. If this failed, we used thrombostatic sealant as a next step. However, high-pressure vessels, such as systemic arteries, are not easy to repair using sealant, whereas low-pressure vessels, such as the pulmonary artery, are suitable for repair using a sealant. Yamashita et al. reported that applying a sealant to a subclavian arterial injury resulted in a pseudoaneurysm (10). Therefore, they suggested that the use of a thrombostatic sealant for repair should be limited to low-pressure vessels only.

In this study, 8 of the 40 patients required conversion to a thoracotomy due to significant intraoperative bleeding. Other reports described their own steps to conversion when significant bleeding requiring conversion during a thoracoscopic pulmonary resection was encountered $(10,13)$. Fournel et al. initially increased the number of ports and treated vessel injuries thoracoscopically (13). If this failed, they finally converted to a thoracotomy. Yamashita et al. enlarged the access incision up to $8 \mathrm{~cm}$, which was considered a mini-thoracotomy (10). Subsequently, they enlarged the skin incision depending on the requirements. In our department, when we struggle to achieve hemostasis using thrombostatic sealant thoracoscopically or when intraoperative blood loss reaches $600 \mathrm{~mL}$, we convert the thoracoscopic approach to a thoracotomy, because primary closure with a vascular clamp may be necessary in that situation. It is dangerous to perform a primary closure with a vascular clamp thoracoscopically when accidental massive bleeding has occurred. Moreover, it is more important to treat intraoperative massive bleeding appropriately to avoid it becoming catastrophic. Therefore, enlarging the access incision up to $10-15 \mathrm{~cm}$ is the best first step, and subsequently, the incision can be enlarged further depending on the situation.

Significant differences were observed in most of the perioperative results, except for the occurrence rate of significant bleeding between patients undergoing thoracoscopic anatomic pulmonary resection during the 
first year (April 2012 to March 2013; n=38) and the last year (April 2017 to March 2018; n=73). These results imply that the risk of a vascular injury when a thoracoscopic approach is used is unavoidable even if the surgeon has experience with the thoracoscopic approach. Other previous studies also reported that vascular hazards are inherent in the thoracoscopic approach, regardless of the surgeon's experience $(10,13)$. Therefore, a thoracic surgeon should keep the risk of vascular injury in mind when using the thoracoscopic approach.

\section{Conclusions}

Thoracoscopic anatomic pulmonary resection is feasible and safe if the surgeon performs appropriate hemostasis, although vascular hazards may be inherent during thoracoscopic anatomic pulmonary resection regardless of the surgeon's experience. Applying sealants and a compression technique using a cotton stick or adjacent lung parenchyma are important to achieve hemostasis in the event of significant vessel injury during a thoracoscopic anatomic pulmonary resection.

\section{Acknowledgments}

The authors appreciate the contributions of all of the surgeons and coworkers who participated in this study and thank the editors and reviewers for their help with this manuscript.

\section{Footnote}

Conflicts of Interest: Meeting presentation: the abstract was presented at the Clinical/Outcomes, Cardiothoracic and Vascular QuickShot Session, American Surgical Congress, Houston, TX, USA, on 7 February 2019.

Ethical Statement: The authors are accountable for all aspects of the work and will ensure that questions related to the accuracy or integrity of any part of the work are appropriately investigated and resolved. This study was approved by the Japanese Red Cross Maebashi Hospital Review Board (No. 2019-40); all patients provided written informed consent prior to their inclusion in the study.

\section{References}

1. Stephens N, Rice D, Correa A, et al. Thoracoscopic lobectomy is associated with improved short-term and equivalent oncological outcomes compared with open lobectomy for clinical Stage I non-small cell lung cancer: a propensity-matched analysis of 963 cases. Eur J Cardiothorac Surg 2014;46:607-13.

2. Jeon JH, Kang CH, Kim HS, et al. Video-assisted thoracoscopic lobectomy in non-small-cell lung cancer patients with chronic obstructive pulmonary disease is associated with lower pulmonary complications than open lobectomy: a propensity score-matched analysis. Eur J Cardiothorac Surg 2014;45:640-5.

3. Paul S, Sedrakyan A, Chiu YL, et al. Outcomes after lobectomy using thoracoscopy vs thoracotomy: a comparative effectiveness analysis utilizing the Nationwide Inpatient Sample database. Eur J Cardiothorac Surg 2013;43:813-7.

4. Scott WJ, Allen MS, Darling G, et al. Video-assisted thoracic surgery versus open lobectomy for lung cancer: A secondary analysis of data from the American College of Surgeons Oncology Group Z0030 randomised clinical trial. J Thorac Cardiovasc Surg 2010;139:976-81.

5. Igai H, Kamiyoshihara M, Kawatani N, et al. Thoracoscopic caudal left lower lobectomy in a patient with fused fissure. Asian J Endosc Surg 2014;7:342-4.

6. Solaini L, Prusciano F, Bagioni P, et al. Video-assisted thoracic surgery (VATS) of the lung: Analysis of intraoperative and postoperative complications over 15 years and review of the literature. Surg Endosc 2008;22:298-310.

7. Sawada S, Komori E, Yamashita M. Evaluation of videoassisted thoracoscopic surgery lobectomy requiring emergency conversion to thoracotomy. Eur J Cardiothorac Surg 2009;36:487-90.

8. Liang C, Wen H, Guo Y, et al. Severe intraoperative complications during VATS lobectomy compared with thoracotomy lobectomy for early stage non-small cell lung cancer. J Thorac Dis 2013;5:513-7.

9. Mei J, Pu Q, Liao H, et al. A novel method for troubleshooting vascular injury during anatomic thoracoscopic pulmonary resection without conversion to thoracotomy. Surg Endosc 2013;27:530-7.

10. Yamashita S, Tokuishi K, Moroga T, et al. Totally thoracoscopic surgery and troubleshooting for bleeding in non-small cell lung cancer. Ann Thorac Surg 2013;95:994-9.

11. Miyazaki T, Yamasaki N, Tsuchiya T, et al. Management of unexpected intraoperative bleeding during thoracoscopic pulmonary resection: a single institutional experience. 
Surg Today 2016;46:901-7.

12. Flores RM, Ihekweazu U, Dycoco J, et al. Video-assisted thoracoscopic surgery (VATS) lobectomy: catastrophic intraoperative complications. J Thorac Cardiovasc Surg 2011;142:1412-7.

13. Fournel L, Zaimi R, Grigoroiu M, et al. Totally thoracoscopic major pulmonary resections: an analysis of perioperative complications. Ann Thorac Surg

Cite this article as: Igai $\mathrm{H}$, Kamiyoshihara M, Yoshikawa R, Ohsawa F, Yazawa T, Matsuura N. Algorithm-based troubleshooting to manage bleeding during thoracoscopic anatomic pulmonary resection. J Thorac Dis 2019;11(11):45444550. doi: $10.21037 /$ jtd.2019.10.72
2014;97:419-24.

14. Igai H, Kamiyoshihara M, Yoshikawa R, et al. Management of intraoperative bleeding during thoracoscopic pulmonary resection in Japan. J Vis Surg 2018;4:225.

15. Igai H, Kamiyoshihara M, Ibe T, et al. Troubleshooting for bleeding in thoracoscopic anatomic pulmonary resection. Asian Cardiovasc Thorac Ann 2017;25:35-40. 